\title{
The Effects of Boron-Doping on the Electronic Properties of Blue Phosphorene
}

\author{
Yejin Wu, Kexin Ma, Zhiyong Wang, Xueqiong Dai* \\ College of Science, Guilin University of Technology, Guilin, China \\ Email: ^daisy@glut.edu.cn
}

How to cite this paper: Wu, Y.J., Ma, K.X., Wang, Z.Y. and Dai, X.Q. (2021) The Effects of Boron-Doping on the Electronic Properties of Blue Phosphorene. Graphene, 10, 41-47.

https://doi.org/10.4236/graphene.2021.103003

Received: May 15, 2021

Accepted: June 15, 2021

Published: June 18, 2021

Copyright $\odot 2021$ by author(s) and Scientific Research Publishing Inc. This work is licensed under the Creative Commons Attribution International License (CC BY 4.0).

http://creativecommons.org/licenses/by/4.0/

\begin{abstract}
In this paper, the effects of different boron (nitrogen)-doping on the electronic properties of blue phosphorene have been investigated by the firstprinciples calculations. We have taken eight doping configurations into account, the calculated results show that the bond length of P-B is decreasing with the doping concentration increasing. For the four boron atoms doping configuration, the geometric structure appears the distinct distortion. The band gap is decreasing with the doping concentration increasing, and it appears the transition from indirect band gap to direct band gap for boron doping configurations. It is hoped that the calculated results may be useful for designing electronic devices based on blue phosphorene.
\end{abstract}

\section{Keywords}

Blue Phosphorene, Boron-Doping, Band Gap

\section{Introduction}

A lot of researchers have investigated two-dimensional materials (2D) owing to the appearance of graphene [1] [2]. However, the zero band gap of graphene will hinder the development of graphene-based electronic device [3] [4]. A new two-dimensional material, blue phosphorene has high carrier mobility [5], modulational bandgaps [6], which can be fabricated experimentally by molecular-beam epitaxial growth [7] [8] [9] [10]. Meanwhile, it can be found that blue phosphorene also exists some faults such as large band gap, non-magnetic, and indirect band gap, which hinder potential applications on optical electronics, spintronics and so on [11] [12] [13]. Bahar Meshginqalam et al. have investigated vacancy defected blue and black phosphorene nanoribbons as gas sensor of NOx and SOx molecules, it can be found that the zigzag phosphorene nanoribbon appears the great current modifications before and after sensing process 
[14]. Daughty John et al. have studied the effects of point defects on the electronic structure and hydrogen storage capability, the calculated results show that defective blue phosphorene can store more hydrogen than black phosphorene [15]. R.M. Arif Khalil et al. have investigated the effects of dopants and vacancy on the optoelectronic, magnetic and dynamical properties of blue phosphorene, it can be found that the different dopants can tune the band gap of blue phosphorene [16]. Xiang Xiao et al. have studied the potential application of black and blue phosphorene as cathode materials in rechargeable aluminum batteries by using the first-principles, it appears a semiconducting-to-metallic transition in the $\left(\mathrm{AlCl}_{4}\right)_{8} \mathrm{P}_{16}$ compound, and the band gap can be tuned [17]. Xiang-Qian Lu et al. have investigated the energetic, electronic, magnetic, and spin-resolved transport properties of hydrogenated armchair and zigzag blue phosphorene nanoribbons with surface modification by $3 \mathrm{D}$ transition metal atoms, it can be found that the magnetic properties can be tuned with different atoms adsorption [18].

Up to now, the effects of different Boron-doping concentration on blue phosphorene have not been investigated systematically. So in this paper, we will investigate the effects of different boron-doping on the electronic properties of blue phosphorene by using first-principles calculations. It is suggesting that the calculated results should be good for designing blue phosphorene-based devices.

\section{Calculation Method}

The geometric structures and electronic properties of blue phosphorene with or without Boron-doping are studied by using the Spanish Initiative for Electronic Simulations with Thousands of Atoms (SIESTA-3.2) [19] [20] [21]. We have considered the exchange correction potential by means of the generalized gradient approximation (GGA) in the form of Perdew and Burke and Ernzerhof (PBE) [22]. A double- $\xi$ plus polarization function (DZP) atomic orbital basis set is used in this paper. $200 \mathrm{Ry}$ for plane cutoff energy and $10^{-4} \mathrm{eV}$ for the energy convergence criteria are chosen during the structural relaxation and calculation of properties, and a vacuum region of the $\mathrm{Z}$ direction is larger than $10 \AA$ in order to eliminate the interaction. The $5 \times 5 \times 1$ supercell of blue phosphorene contains 50 phosphorus atoms (as shown in Figure 1).

\section{Results and Discussions}

In this paper, the geometric and electronic properties of blue phosphorene with or without boron-doping are studied, $\mathrm{P}$ configuration refers to the perfect blue phosphorene (as shown in Figure 1), B1 refers to one boron atom doping configuration, B3 refers to three boron atoms doping configuration, B4 refers to four boron atoms doping configuration, B6 refers to six boron atoms doping configuration. For the $\mathrm{P}$ configuration, all the bond length are $2.292 \AA$. For the $\mathrm{B} 1$ configuration, the formed $\mathrm{P}-\mathrm{B}$ bond are shorter than the counterpart of $\mathrm{P}$ configuration, and the shortest P-B bond length is $1.939 \AA$. For B3 configuration, the shortest P-B bond length is $1.898 \AA$. For $\mathrm{B} 4$ configuration, the shortest 
P-B bond length is $1.756 \AA$. For B6 configuration, the shortest P-B bond length is $1.735 \AA$. It can be found that it appears the distortion owing to boron-doping, which may be attributed to the stronger electronegativity of boron atom (Figure 2).

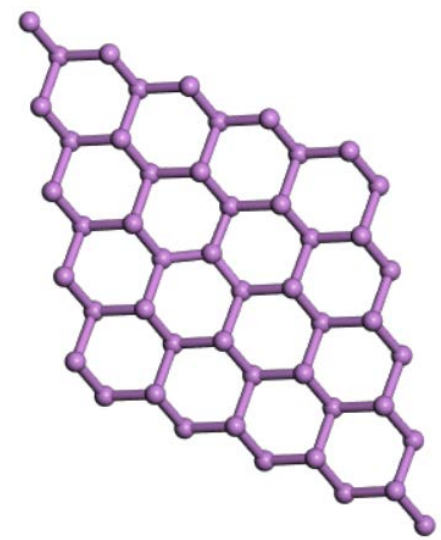

Figure 1. The model of perfect blue phosphorene (P configuration).

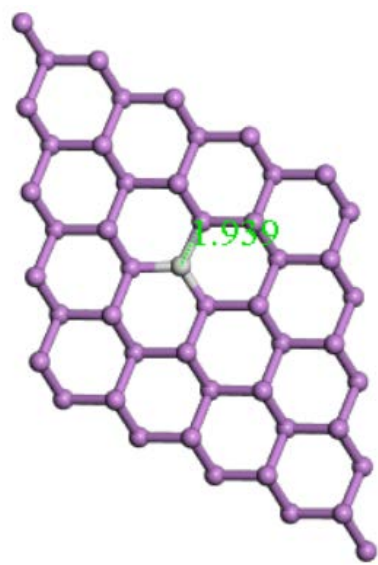

(a)

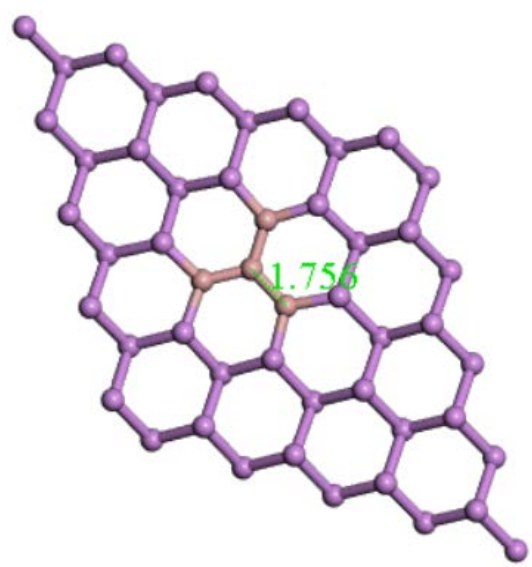

(c)

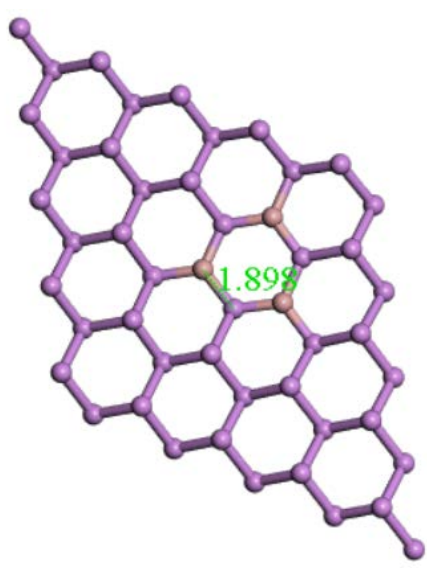

(b)

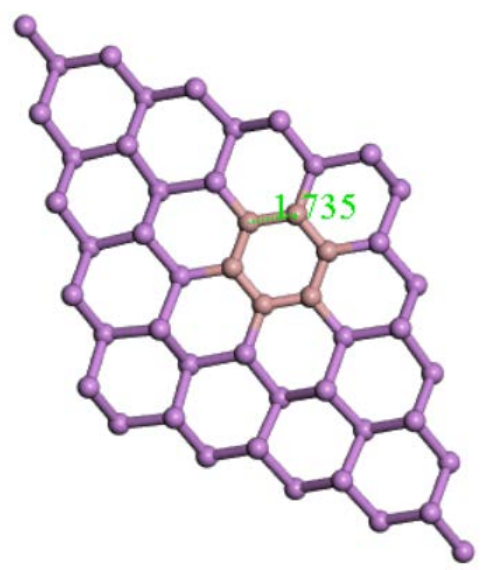

(d)

Figure 2. The different boron-doping blue phosphorene. (a), (b), (c), (d) refer to B1, B3, B4, B6 configuration, respectively. 
The change of geometric structure can affect the electronic property of blue phosphorene. And we will take the electronic properties of blue phosphorene with or without boron-doping into account (as shown in Figure 3), the band gap of $\mathrm{P}$ configuration is $2.02 \mathrm{eV}$, and the band gap of $\mathrm{B} 1, \mathrm{~B} 3, \mathrm{~B} 4$ and $\mathrm{B} 6$ is $1.64 \mathrm{eV}$, $1.64 \mathrm{eV}, 0.59 \mathrm{eV}$ and $0.58 \mathrm{eV}$, respectively. It can be found that the band gap of blue phosphorene can be tuned by modulating the boron-doping concentration. Interestingly, it appears the transition from indirect band gap to direct band gap, and it can be found that the impurity subbands appear in the valance band near the fermi level, which can be attributed to the distortion of blue phosphorene owing to the different boron-doping, it may be useful for designing the optoelectronics devices based on blue phosphorene.

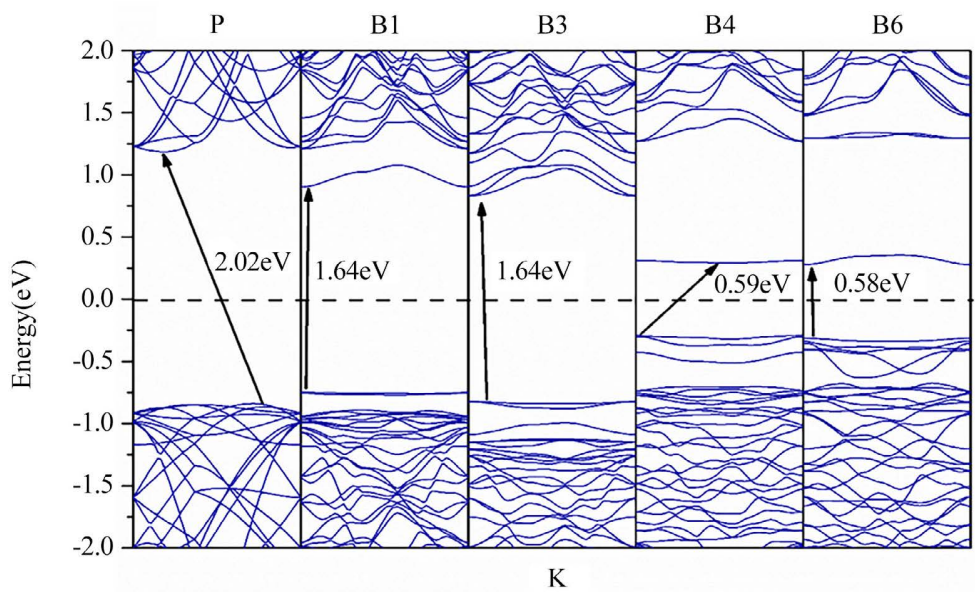

Figure 3. The band structures of blue phosphorene, $\mathrm{P}$ refers to perfect blue phosphorene, B1, B3, B4, B6 refer to different boron-doping configurations, respectively. The wave vector $\mathrm{K}$ is from $\Gamma$ to $\mathrm{X}$. The energy at Fermi level is set to zero and shown as the dashed line.

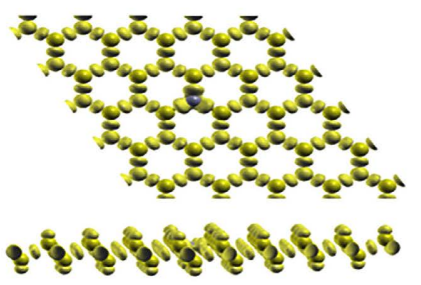

(a)

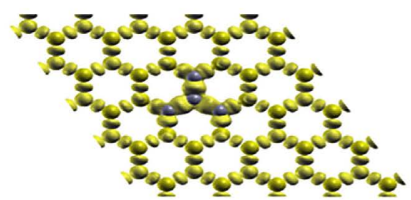

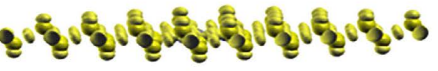

(c)
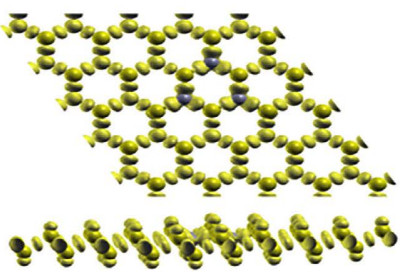

(b)

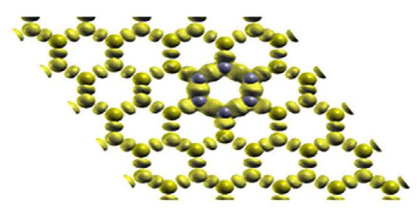

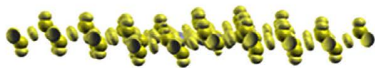

(d)

Figure 4. The charge density of blue phosphorene with different boron-doping. (a), (b), (c), (d) refer to B1, B3, B4 and B6 configuration, respectively. 
Next, we will continue to investigate the charge densities of blue phosphorene with different boron-doping (as shown in Figure 4). It can be found that the charge density near the boron atom is distinctly increasing. In B1 configuration, the boron atom attracts 0.141 electrons from the orbitals of the nearest phosphorous atoms. In $\mathrm{B} 3$ configuration, the boron atoms attracts 0.834 electrons from the orbitals of the nearest phosphorous atoms. In B4 configuration, the boron atoms attract 0.423 electrons from the orbitals of the nearest phosphorous atoms. In B6 configuration, the boron atoms attract 0.704 electrons from the orbitals of the nearest phosphorous atoms.

\section{Conclusion}

In this paper, the geometric and electronic properties of blue phosphorene with or without boron-doping are investigated by using the first-principles. The bond length of P-B bond is shorter than that of P-P bond of perfect blue phosphorene owing to the stronger electronegativity of boron atom. The different boron-doping configurations can tune the band gap of blue phosphorene, and it appears the corresponding charge transfer. It is hoped that our calculated results may be useful for designing the electronic device based on blue phosphorene.

\section{Acknowledgements}

This paper is supported by the Middle-aged and Young Teachers' Basic Ability Promotion Project of Guangxi (Grant No. 2021KY0267).

\section{Conflicts of Interest}

The authors declare no conflicts of interest regarding the publication of this paper.

\section{References}

[1] Novoselov, K.S., Jiang, D., Schedin, F., Booth, T.J., Khotkevich, V., Morozov, S.V. and Geim, A.K. (2005) Two Dimensional Atomic Crystals. Proceedings of the National Academy of Sciences of the United States of America, 102, 10451-10453. https://doi.org/10.1073/pnas.0502848102

[2] Zhang, H.-P., Kou, L., Jiao, Y., Du, A., Tang, Y. and Ni, Y. (2020) Strain Engineering of Selective Chemical Adsorption on Monolayer Black Phosphorous. Applied Surface Science, 503, Article ID: 144033.

https://doi.org/10.1016/j.apsusc.2019.144033

[3] Silvestrelli, P.L. and Ambrosetti, A. (2018) Bandgap Opening in Graphene Using Alkali Ions by First Principles. Applied Physics Letters, 113, Article ID: 211603. https://doi.org/10.1063/1.5060660

[4] Ambrosetti, A. and Silvestrelli, P.L. (2019) Cooperative Effects of N-Doping and $\mathrm{Ni}(111)$ Substrate for Enhanced Chemical Reactivity of Graphene: The Case of CO and $\mathrm{O}_{2}$ Adsorption. The Journal of Physical Chemistry C, 123, 31050-31056. https://doi.org/10.1021/acs.jpcc.9b08918

[5] Xiao, J., Long, M.Q., Zhang, X.J., Ouyang, J., Xu, H. and Gao, Y.L. (2015) Theoretical Predictions on the Electronic Structure and Charge Carrier Mobility in 2D 
Phosphorene Sheets. Scientific Reports, 5, Article No. 9961. https://doi.org/10.1038/srep09961

[6] Chowdhury, C., Jahiruddin, S. and Datta, A. (2016) Pseudo-Jahn-Teller Distortion in Two-Dimensional Phosphorene: Origin of Black and Blue Phases of Phosphorene and Band Gap Modulation by Molecular Charge Transfer. The Journal of Physical Chemistry Letters, 7, 1288-1297. https://doi.org/10.1021/acs.jpclett.6b00219

[7] Zhu, Z. and Tománek, D. (2014) Semiconducting Layered Blue Phosphorene: A Computational Study. Physical Review Letters, 112, Article ID: 176802. https://doi.org/10.1103/PhysRevLett.112.176802

[8] Zhang, J.L., Zhao, S.T., Han, C., Wang, Z.Z., Zhong, S., Sun, S., Guo, R., Zhou, X., Gu, C.D., Yuan, K.D., Li, Z.Y. and Chen, W. (2016) Epitaxial Growth of Single Layer Blue Phosphorene: A New Phase of Two-Dimensional Phosphorene. Nano Letters, 16, 4903-4908. https://doi.org/10.1021/acs.nanolett.6b01459

[9] Xu, J.-P., Zhang, J.-Q., Tian, H., Xu, H., Ho, W. and Xie, M. (2017) One Dimensional Phosphorene Chain and Two-Dimensional Blue Phosphorene Grown on $\mathrm{Au}(111)$ by Molecular-Beam Epitaxy. Physical Review Materials, 1, Article ID: 061002. https://doi.org/10.1103/PhysRevMaterials.1.061002

[10] Han, N., Gao, N. and Zhao, J. (2017) Initial Growth Mechanism of Blue Phosphorene on $\mathrm{Au}(111)$ Surface. The Journal of Physical Chemistry C, 121, 17893-17899. https://doi.org/10.1021/acs.jpcc.7b04209

[11] Villegas, C.E.P., Rodin, A.S., Carvalho, A. and Rocha, A.R. (2016) Two-Dimensional Exciton Properties in Monolayer Semiconducting Phosphorene Allotropes. Physical Chemistry Chemical Physics, 18, 27829-27836. https://doi.org/10.1039/C6CP05566D

[12] Hugh, C.O.H. and Pablo, J.H. (2014) Two-Dimensional Crystals: Phosphorus Joins the Family. Nature Nanotechnology, 9, 330-331. https://doi.org/10.1038/nnano.2014.85

[13] Xu, W., Zhao, J. and $\mathrm{Xu}, \mathrm{H}$. (2018) Adsorption Induced Indirect-to-Direct Band Gap Transition in Monolayer Blue Phosphorene. The Journal of Physical Chemistry C, 122, 15792-15798. https://doi.org/10.1021/acs.jpcc.8b05125

[14] Meshginqalam, B. and Barvestani, J. (2020) Vacancy Defected Blue and Black Phosphorene Nanoribbons as Gas Sensor of $\mathrm{NO}_{x}$ and $\mathrm{SO}_{x}$ Molecules. Applied Surface Science, 526, Article ID: 146692. https://doi.org/10.1016/j.apsusc.2020.146692

[15] John, D., Nharangatt, B., Kastuar, S.M. and Chatanathodi, R. (2021) Blue Phosphorene Nanosheets with Point Defects: Electronic Structure and Hydrogen Storage Capability. Applied Surface Science, 551, Article ID: 149363.

https://doi.org/10.1016/j.apsusc.2021.149363

[16] Arif Khalil, R.M., Hussain, F., Hussain, M.I., Parveen, A., Imran, M., Murtaza, G., Sattar, M.A., Rana, A.M. and Kim, S. (2020) The Investigation of Optoelectronic, Magnetic and Dynamical Properties of Ce and Ti Doped 2D Blue Phosphorene: A Dispersion Corrected DFT Study. Journal of Alloys and Compounds, 827, Article ID: 154255. https://doi.org/10.1016/j.jallcom.2020.154255

[17] Xiao, X., Wang, M.Y., Tu, J.G. and Jiao, S.Q. (2019) The Potential Application of Black and Blue Phosphorene as Cathode Materials in Rechargeable Aluminum Batteries: A First-Principles Study. Physical Chemistry Chemical Physics, 21, 7021. https://doi.org/10.1039/C9CP00453J

[18] Lu, X.Q., Wang, C.K. and Fu, X.X. (2019) Modulating the Electronic Structures of Blue Phosphorene towards Spintronics. Physical Chemistry Chemical Physics, 21, 11755. https://doi.org/10.1039/C9CP01684H 
[19] Ordejón, P., Artacho, E. and Soler, J.M. (1996) Self-Consistent Order-N Density-Functional Calculations for Very Large Systems. Physical Review B, 53, R10441. https://doi.org/10.1103/PhysRevB.53.R10441

[20] Sánchez-Portal, D., Ordejón, P., Artacho, E. and Soler, J.M. (1997) Density Functional Method for Very Large Systems with LCAO Basis Sets. International Journal of Quantum Chemistry, 65, 453-461.

https://doi.org/10.1002/(SICI)1097-461X(1997)65:5<453::AID-QUA9>3.0.CO;2-V

[21] Soler, J.M., Artacho, E., Gale, J.D., García, A., Junquera, J., Ordejón, P. and Sánchez-Portal, D. (2002) The Siesta Method for ab Initio Order-N Materials Simulation. Journal of Physics: Condensed Matter, 14, 2745-2779.

https://doi.org/10.1088/0953-8984/14/11/302

[22] Perdew, J.P., Burke, K. and Ernzerhof, M. (1996) Generalized Gradient Approximation Made Simple. Physical Review Letters, 77, 3865-3868.

https://doi.org/10.1103/PhysRevLett.77.3865 